Signal \& Image Processing : An International Journal (SIPIJ) Vol.5, No.3, June 2014

\title{
AN INTENSITY-BASED MEDICAL IMAGE REGISTRATION USING GENETIC ALGORITHM
}

\author{
Shanmugapriya.S $S^{1}$, Dr.S.Poonguzhali ${ }^{2}$, Umamaheshwari $^{3}$ \\ Department of Medical Electronics, CEG, Anna University, Chennai, India
}

\begin{abstract}
Medical imaging plays a vital role to create images of human body for clinical purposes. Biomedical imaging has taken a leap by entering into the field of image registration. Image registration integrates the large amount of medical information embedded in the images taken at different time intervals and images at different orientations. In this paper, an intensity-based real-coded genetic algorithm is used for registering two MRI images. To demonstrate the efficiency of the algorithm developed, the alignment of the image is altered and algorithm is tested for better performance. Also the work involves the comparison of two similarity metrics, and based on the outcome the best metric suited for genetic algorithm is studied.
\end{abstract}

\section{KEYWORDS}

Image registration, Genetic algorithm, Similarity metric

\section{INTRODUCTION}

Image registration aligns the information in different images and to visualize as a combined image. Image registration operates for different conditions such as images taken at different time, view point and/ or different sensors [3]. The goal of image registration is to find the best geometrical mapping to align the images. This geometrical alignment of medical images has its rapid growth in diagnosis, treatment planning, computer-aided therapies and in biomedical research. Image registration falls under two categories- voxel or intensity - based and feature based. In intensity-based method, the whole image is employed; where they operate directly on image intensity. In feature-based method, features like contours, crest lines, points- have to be extracted from the images in pre-processing step and has to be matched.

Intensity-based method works better than feature-based image registration [1][2]. Image registration techniques generally depends on many criteria such as, nature of registration, ie, extrinsic or intrinsic based, transformation model and optimisation procedure [4] . The most applicable algorithms for medical image registration are based on optimising voxel-based similarity measure. Surface registration techniques focus on three factors, namely, choice of transformation, similarity criterion and global optimisation [6]. Many metrics such as, sum of squared differences, sum of absolute differences, mutual information, normalised correlation can be used for intensity-based medical image registration [7]-[9]. The drawbacks of traditional numerical based optimisation algorithms like, gradient descent, Powell's method, can be 
Signal \& Image Processing : An International Journal (SIPIJ) Vol.5, No.3, June 2014

overcome by genetic algorithm. It finds a global optimal solution, escaping from local optima [5][10].

In this paper, image registration is done by genetic algorithm. The most crucial step in GA is to choose the objective function which is used to evaluate the fitness of each chromosome. The fitness value is determined by the similarity metrics which is the objective function. Here normalised correlation is used as the objective function for GA process. The result obtained by normalised correlation is compared with mutual information method which is estimated by mean squared error.

\section{Methodology}

Fig1 shows the flow of image registration process. Image registration process involves two MRI images, namely, reference image $I_{R}$, and a scene image $I_{S}$, which has to be aligned in accordance with the reference image[1].

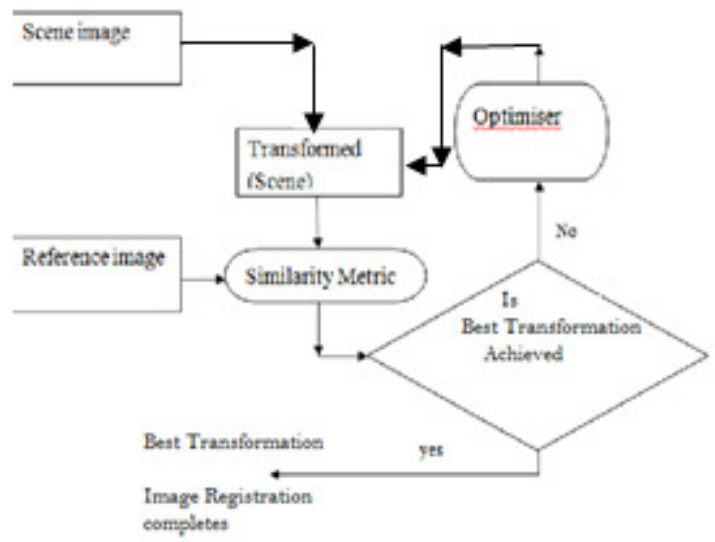

Fig1. Flow of image registration process

The result of registration process is that the reference $I_{R}$ and transformed scene image $f\left(I_{S}\right)$ are similar possible.

Of those two images, one image is rotated to 180 degrees and this is taken as scene image. Similarity transformations, ie, translation, rotation and scaling are applied to scene image. The similarity between the reference and transformed scene image are determined by normalised correlation which is the similarity metric. If $\mathrm{A}(\mathrm{x}, \mathrm{y})$ and $\mathrm{B}(\mathrm{x}, \mathrm{y})$ are two images, Normalised correlation(NC) is defined.

$$
\mathrm{NC}=\frac{1}{n} \sum_{x, y} \frac{(A(x, y)-\bar{A})}{\sigma_{A}} \frac{(B(x, y)-\bar{B}}{\sigma_{B}}
$$

Where $\mathrm{n}=$ number of pixels in two images, $\bar{A}, \bar{B}$ are average intensity values. 
Signal \& Image Processing : An International Journal (SIPIJ) Vol.5, No.3, June 2014

\subsection{Genetic Algorithm}

If the best transformation is not obtained, an optimiser is used to find the optimal transformation. Genetic algorithm is used to find the best transformation. The search strategy used is parameterbased method [1][2]. The crucial step in GA is to encode the parameters into GA chromosomes. Real encoding is used than binary encoding. Efficiency of GA is increased since there is no conversion to binary type and different genetic operators can be used [11]. So the encoding involves the transformation parameters directly. It is represented by five real numbers, namely one rotation parameter $(\tau)$, two translation parameters $(\mathrm{t})$, and two scaling parameters $(\mathrm{s})$. GA cycle follows four process- fitness evaluation, selection, recombination and creation of new population. Initial population is set to 50 chromosomes. Normalised correlation is used to find the similarity for each transformation and evaluation of this objective function called fitness value is calculated for each chromosome in the population. Next step involves selection of fittest individuals. Tournament method requires more execution time and hence Roulette's wheel method is used. Highest fit individuals are selected and genetic operators such as; crossover and mutation are applied to create next generation population. Here arithmetic crossover is used.

$\mathrm{P}=\alpha^{*} \mathrm{~A}+(1.0-\alpha) * \mathrm{~B}$

$\mathrm{Q}=(1.0-\alpha)^{*} \mathrm{~A}+\alpha^{*} \mathrm{~B}$

Where, A, B - individual from the old generation, P, Q - individual from new generation, $\alpha$ is the weight which governs dominant individual in reproduction and it is between 0 and 1 .

So the succeeding generations will contain the offspring and also the best fit individuals from its preceding generations. Mutation operators randomly pick one of the individual in the chromosome and replace it by a random value in its range. Fitness value is calculated for the next generation individuals. This process is continued for many generations. Stopping criteria chosen is maximum generation. It is likely that as generation increases, best fitness, i.e. best transformation can be obtained. The process is continued until fitness value reaches high so that the registration completes. The global optimum is achieved by creating new population from individuals of existing population.

\section{EXPERIMENTAL STUDY}

For this experiment, simulated real world MRI images were taken from a public database. Images were obtained from brain web database at McGill University, which can be easily used to evaluate the performance of various image analysis methods. It has been frequently used by biomedical research community. The images taken are with and without noises, and presence or absence of lesions. The images are at the size of $181 \times 217$ pixels.

Table 1: Types of images used

\begin{tabular}{|c|c|c|}
\hline Cases & Reference image & Scene image \\
\hline I & Normal image & $\begin{array}{c}\text { Normal image with 180 } \\
\text { degree rotated }\end{array}$ \\
\hline II & Normal image & Lesion with 1\% noise \\
\hline III & Normal image & Lesion with 4\% noise \\
\hline IV & Normal image & Normal with noise 3\% \\
\hline
\end{tabular}


Signal \& Image Processing : An International Journal (SIPIJ) Vol.5, No.3, June 2014

\subsection{Parameter settings}

Transformation parameters are applied on the pair of images. The translation parameter, rotation parameter and scaling parameter are noted as $t_{\mathrm{x}}, \mathrm{t}_{\mathrm{y}}, \tau, \mathrm{S}_{\mathrm{x}}, \mathrm{S}_{\mathrm{y}}$ respectively. Rotation is taken as 0 to $2 \pi$, translation is taken as [100 300] and scaling as [0.5 2].

Initial population was taken as 50 . Crossover probability is 0.6 and mutation probability is 0.3 . Stopping criteria is taken as the maximum generation. Maximum of 300 generations were done.

\subsection{Analysis of results}

Two pair of MRI images was taken. For each pair of images, GA was performed with NC and with mutual information (MI). Fitness using $\mathrm{NC}$ is calculated by maximising the similarity metric. For each generations, maximum of 10 runs were done. The results obtained are estimated by mean square error (MSE).GA with NC showed better result than GA with MI.

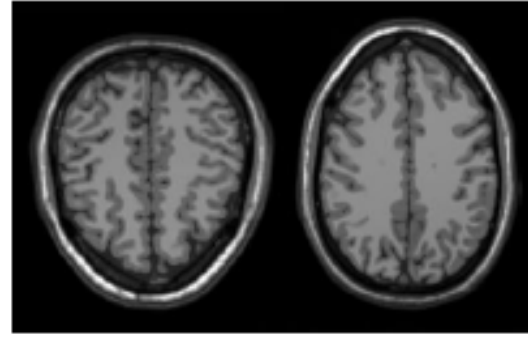

Fig 2(a)

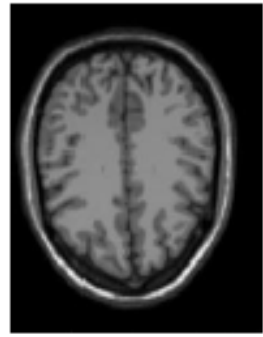

Fig 2(b)

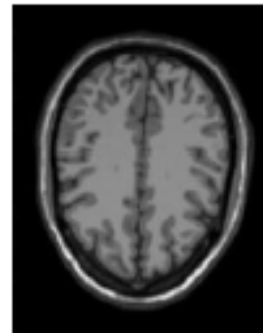

Fig 2(c)

Fig 2(a) represents reference and scene image rotated to 180 degrees. Fig 2(b) represents registered image using NC. Fig 2(c) represents registered image using MI. Fig 2(d) shows the registered image using MSE. The output images shown are obtained in $300^{\text {th }}$ generation
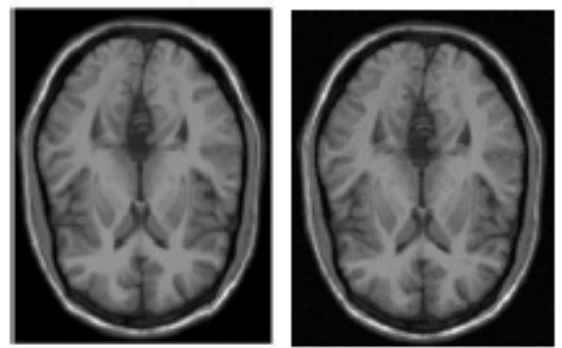

Fig 3(a)

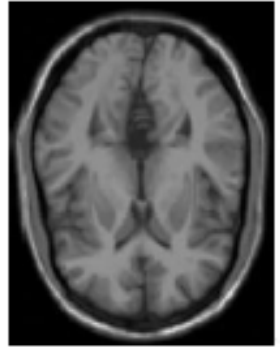

Fig 3(b)

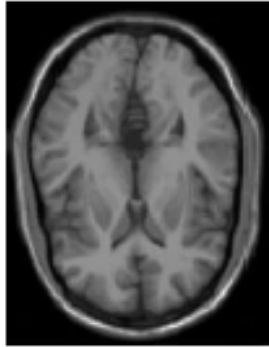

Fig 3(c)

Fig 3(a) represents reference image with no noise and scene image with lesion $1 \%$ noise. Fig 3(b) represents the registration image obtained using NC. Fig 3(c) shows the registration image using MI. The results obtained were taken at 300th generation 
Signal \& Image Processing : An International Journal (SIPIJ) Vol.5, No.3, June 2014

Table 2: Optimisation result using GA based on NC

\begin{tabular}{|c|c|c|c|c|}
\hline Cases & NC FV & Rotation & Translation & Scaling \\
\hline I & 1.906 & 3.14 & {$[177.89153 .00]$} & {$[11]$} \\
\hline II & 1.963 & 6.28 & {$\left[\begin{array}{lll}178 & 153.72\end{array}\right]$} & {$[1]$} \\
\hline III & 1.954 & 6.28 & {$[177.87154 .26]$} & {$[11]$} \\
\hline IV & 1.894 & 6.27 & {$[177.45153 .00]$} & {$[11]$} \\
\hline
\end{tabular}

Table 3: Optimisation result using GA based on MI

\begin{tabular}{|c|c|c|c|c|}
\hline Cases & MI FV & Rotation & Translation & Scaling \\
\hline I & 1.456 & 3.13 & {$\left[\begin{array}{lll}175.00 & 152.21]\end{array}\right.$} & {$\left[\begin{array}{lll}1 & 1\end{array}\right]$} \\
\hline II & 1.729 & 6.27 & {$\left[\begin{array}{llll}178.00 & 153.72]\end{array}\right.$} & {$\left[\begin{array}{lll}1 & 1\end{array}\right]$} \\
\hline III & 1.698 & 6.27 & {$\left[\begin{array}{lll}177.31 & 153.89\end{array}\right]$} & {$\left[\begin{array}{ll}1 & 1\end{array}\right]$} \\
\hline IV & 1.685 & 6.27 & {$\left[\begin{array}{lll}175.13 & 154\end{array}\right]$} & {$\left[\begin{array}{lll}0.99 & 0.99\end{array}\right]$} \\
\hline
\end{tabular}

Table 4 : Comparison of NC and MI using MSE

\begin{tabular}{|c|c|c|}
\hline Cases & NC-MSE & MI-MSE \\
\hline I & 8.193 & 10.988 \\
\hline II & 8.274 & 11.78 \\
\hline III & 8.110 & 9.634 \\
\hline IV & 8.540 & 10.324 \\
\hline
\end{tabular}

\section{CONCLUSION}

It can be seen that in both the cases, normalised correlation showed better convergence and also the MSE value to be less when compared with MI. It is observed that as generations' increases, the fitness value increases. Higher the fitness value and more constant it is, better the convergence.

The objective functions were evaluated for GA using normalised correlation, mutual information and mean square error method. Normalised correlation method shows better performance than the other. Registration is nothing but the calculation of an image transformation $\mathrm{T}$, achieved by optimisation of some similarity measure computed directly from intensity values of the images. In future work, performance of intensity-based and feature- based methods may be analysed.

\section{REFERENCES}

[1] Andrea Valsecchi and Sergio Damas,(2012) “ An Image Registration Approach using Genetic Algorithms", IEEE World Congress on Computational Intelligence.

[2] Andrea Valsecchi and Sergio Damas, Linda Marrakchi-Kacem,(2013) " Genetic Algorithms for Voxel-based Medical Image Registration",IEEE, CIMI,pp. 4673-5919

[3] Barbara Zitova,Jan Flusser,(2003) "Image registration methods: a survey", Image and Vision Computing 21 ,pp. 977-1000.

[4] Derek L G Hill, Philipp G Batchelor,(2001) "Medical image registration”, Phys. Med. Biol.46,pp.145 
Signal \& Image Processing : An International Journal (SIPIJ) Vol.5, No.3, June 2014

[5] Mahua Bhattacharya And Arpita Das,(2007) "Multi Resolution Medical Image Registration Using Maximization Of Mutual Information And Optimization By Genetic Algorithm",IEEE Nuclear science symposium, conference record, pp. 2961-2964

[6] Michel A.Audette, Frank P. Ferrie,(2000) "An Algorithmic Overview Of Surface Registration Techniques For Medical Imaging”, Med Image Anal,vol 4,pp. 201-217

[7] Myung-Eun Lee, Soo-Hyung Kim,(2009) "Intensity-Based Registration Of Medical Images", International conference on test and measurement,pp.239-242

[8] C.Sasi varnan, A.Jagan, Jaspreet Kaur, Divya Jyoti, Dr.D.S.Rao,(2011) "Image Quality Assessment Techniques on Spatial Domain", IJCST, Vol. 2, Issue 3,pp. 177-184.

[9] J. N. Ulysses, A. Conci,(2010) "Measuring Similarity in Medical Registration", IWSSIP 17th International Conference on Systems, Signals and Image Processing.

[10] Xiaoguang Pan, Kai Zhao, Jiren Liu, Yan Kang,(2010) "A Hybrid Optimization Method For Maximum Mutual Information Registration", International conference on biomedical engineering and informatics,pp.18-22

[11] Yalcinoz T, Altun H and Uzam M,(2001) "Economic dispatch solution using a genetic algorithm based on arithmetic crossover." IEEE Porto PowerTech',Porto, Portugal, 10-13 September 2001.Paper No. AIT1-166.

\section{AUTHORS}

Shanmugapriya.S completed B.E- Biomedical engineering in Rajiv Gandhi College of Engineering. Chennai. Currently doing M.E - Medical Electronics in College of Engineering, Guindy, Anna University, Chennai. Field of interest are image processing, tissue engineering and bio instrumentation.

Dr.S.Poonguzhali received her $\mathrm{PhD}$ degree (Medical Image Processing) from Anna University, Chennai in 2009. She has 17 years of experience in teaching and also has published more than 70 papers in National and International Journals and conference. Her research interest is Biomedical Instrumentation, Medical Image Processing. Currently, she is working as Assistant Professor in Department of ECE, College of Engineering, Guindy, Anna University, Chennai.

Ms. Umamaheshwari, Research Scholar in Anna University, has published many papers in National conference. Her research interest is Medical Image Processing 